\title{
Magnetic Design and Simulation Analysis of Magneto-rheological Damper
}

\author{
Xiaojuan $\mathrm{Li}^{1, \mathrm{a}}$, Xiaobin Liang ${ }^{1, \mathrm{~b}}$, Fan $\mathrm{He}^{1, \mathrm{c}}$, Wei Guo ${ }^{1, \mathrm{~d}}$, Weibin Wang ${ }^{1, \mathrm{e}}$ \\ ${ }^{1}$ Communication \& Power Utilization Subcomany of Nari Group Corporation, Haidian, Beijing, \\ 102200, China \\ alixiaojuan2@sgepri.sgcc.com.cn, ${ }^{b}$ liangxiaobin@sgepri.sgcc.com.cn, \\ chefan1@sgepri.sgcc.com.cn, ${ }^{d}$ guowei7@sgepri.sgcc.com.cn, \\ ewangweibin@sgepri.sgcc.com.cn
}

Keywords: MR; Damper; Magnetic Design; Simulation

\begin{abstract}
Magneto-rheological damper has advantages of fast response, good stability and continuously adjustable damping force, that is controlled by the damping force to achieve damping. This paper analyzes the influence of the thickness of the outer cylinder, work space, turns, damping channel length, coil currents and other factors on magnetic field intensity in damping channel by ANSYS software, it provides theoretical basis for the overall structural design of MR damper.
\end{abstract}

\section{Introduction}

Magneto-rheological fluid ${ }^{[1]}$ is referred as MRF, which is a kind of suspension in a kid of carrier liquid from in a large number of magnetizable particles which constitute its rheological properties change with the applied magnetic field changes.

Since $1980^{\text {th }}$, the Magneto-rheological technology has been developing rapidly, researches on MR Damping Equipment ${ }^{[2]}$ resulted in many inspiring achievements which revealed bright prospect for human being. MR Damper is a hot research spot internationally because of its small volume, low power requirements, big damping force, wide dynamic range, high frequency response, wide applying occasions, etc. Domestic researchers are also attaching importance to it gradually. MR damper is widely used in automobile manufacturing, machinery manufacturing industry, weapons systems, helicopters, civil engineering and other fields.

This paper presents some of the key through the magnetic circuit design size Magneto-rheological damper optimization methods to further improve the performance of Magneto-rheological damper.

\section{Principle of MRF Damper}

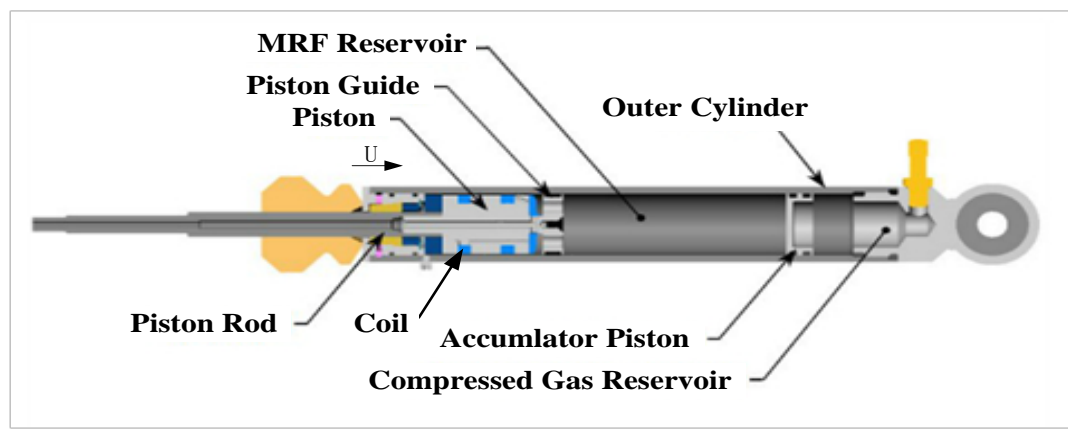

Fig.1 Functional Principle of MR Damper

MR damper can achieve vibration damping by magneto-rheological fluid in the gap generated by the flow of damping force. Fig. 1 indicates MR damper operating principle. The outer cylinder is fixed, the piston moves axially with velocity U, MRF is squeezed flows between the gap of the outer cylinder and the piston, the force generated by the damping can be controlled by the external magnetic field. 


\section{Magnetic Circuit Design of MR Damper}

The main task of the magnetic circuit design is: ensure that the magnetic field lines perpendicular to the gap and fluid work by rational design of the core structure, and the magnetic field strength has the highest concentration of the fluid gap. MR damper magnetic field lines through the area includes four parts which including piston、 piston flank v permeable tube and damping path gap. As shown in Fig.2, magnetic force lines are from the piston I, go through the piston flanking II, then pass the damping path gap IV, enter the permeable tube III, finally pass the gap IV of another part, pass the piston flank II enters I, which form a closed loop. According to the flux continuity theorem, the total magnetic flux is:

$\Phi_{\text {Total }}=\Phi_{\mathrm{I}}=\Phi_{\mathrm{II}}=\Phi_{\mathrm{III}}=\Phi_{\mathrm{IV}}$

The total reluctance of magnetic structure is:

$\mathrm{R}=\mathrm{R}_{\mathrm{I}}+2 \mathrm{R}_{I I}+\mathrm{R}_{\mathrm{III}}+2 \mathrm{R}_{\mathrm{IV}}$

(2)

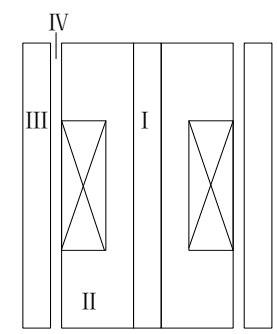

Fig.2 The Magnetic Circuit of MR Damper

I- Piston II- Piston Flank III - Permeable Tube IV - Damping Channel Gap

\section{Magnetic Circuit Simulation of MRF Damper}

ANSYS is a kind of general purpose finite element analysis software, its electromagnetic module is based on Maxwell's equations field guide. It can be used to analyze electromagnetic problems such as inductance, capacitance, impedance, magnetic flux density and magnetic field strength, etc. In this paper, ANSYS finite element analysis software is used to simulate the magnetic field of MR damper field ${ }^{[3]}$, which analyzes the impact of the current intensity, the geometry of the core structure of the magnetic field distribution, etc.

In order to improve the performance of damping force MR damper, the main method is to improve the magnetic field strength of Magneto-rheological fluid work area. Therefore, magnetic circuit analysis is calculating the magnetic field intensity of damping channel.

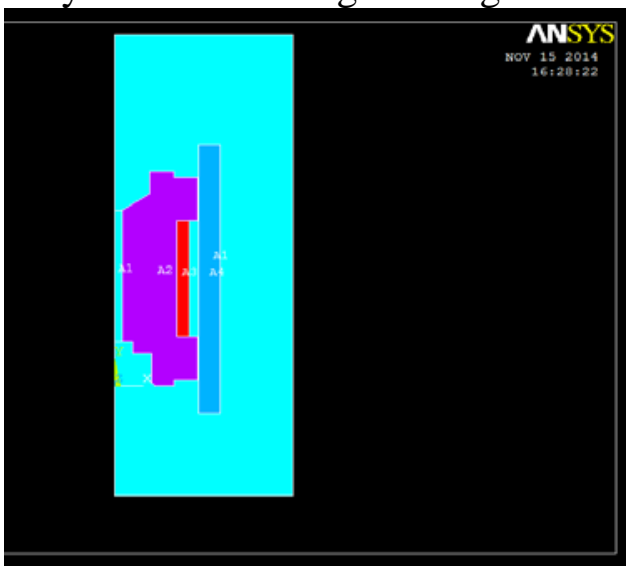

Fig.3 Two-dimensional Analysis Model of Damper Simplified



Fig.4 Magnetic Field Lines Simulation Result

The following is the specific process of MR damper Finite element analysis of the magnetic field strength of the damping channel:

ANSYS product applications; ANSYS / Multiphysics:

Application branches: Magnetism; 
Type of analysis: 2D linear static analysis;

Unit type to use: PLANE 53;

Although the Magneto-rheological fluid damper is a three-dimensional object, but it is symmetry and its structure is simple, this paper uses the 2-D static magnetic field analytical method which is simplified. Fig.3 is analysis model of damper simplified.

Fig.4 is the magnetic field lines simulation result. As can be seen from Fig.5, the magnetic lines of force in the damping channel is substantially equidistant distribution, therefore, this area as a uniform magnetic field approximation is reasonable in ANSYS software.

Fig.5 shows the magnetic field intensity distribution contour, the result indicates the maximum magnetic field intensity distribution is in the damping channel.



Fig.5 Distribution Contour of Magnetic field Intensity Simulation Result

Fig.6 shows the simulation results of different parameters such as outer cylinder, coil current, working gap, damping channel length, outer cylinder material and coil winding direction corresponding to the magnetic field intensity of damper channel.

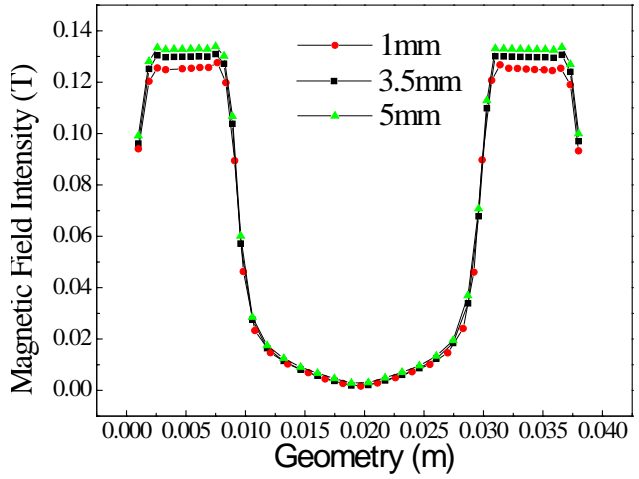

(a) Different Thickness of the Outer Cylinder Corresponding to Magnetic Field Intensity

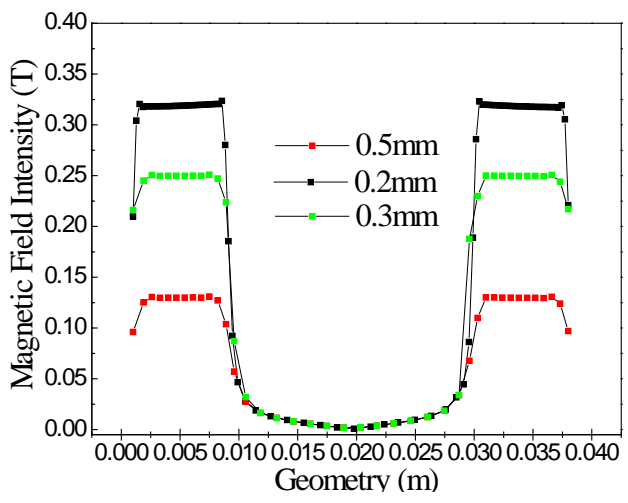

(c) Different Working Gap Corresponding to Magnetic Field Intensity

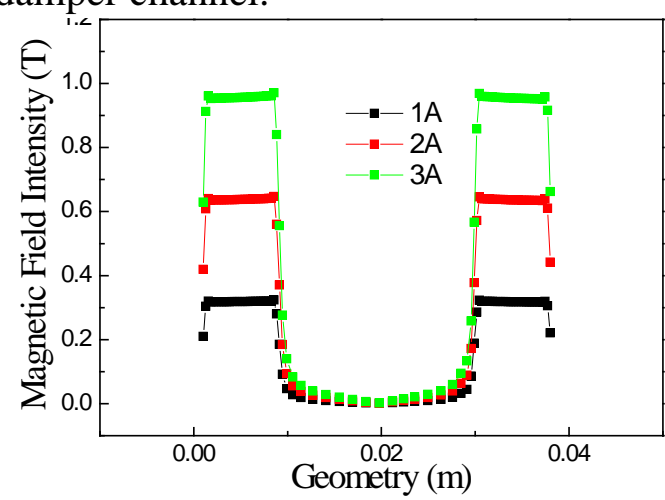

(b) Different Coil Current Corresponding to Magnetic Field Intensity

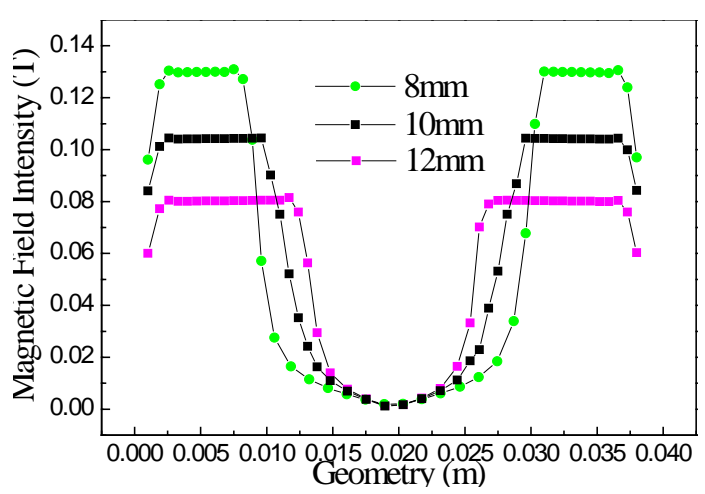

(d) Different Length of Damping Channel Corresponding to Magnetic Field Intensity 


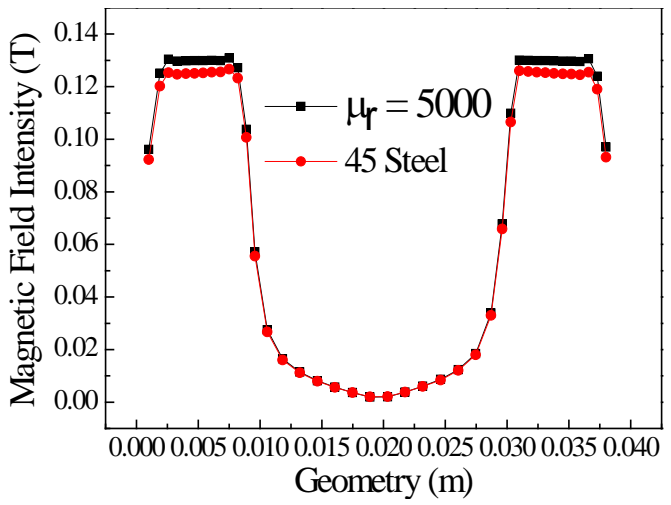

(e) Different Material of the Outer Cylinder Corresponding to Magnetic Field Intensity

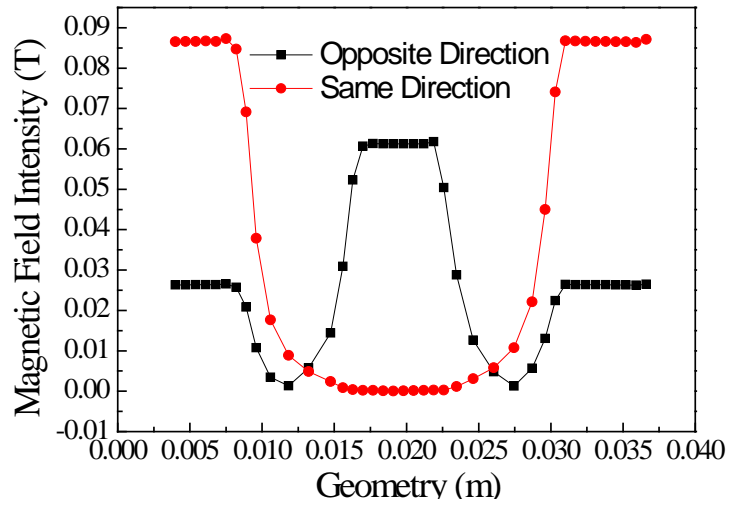

(f) Different Coil Winding Direction Corresponding to Magnetic Field Intensity

Fig.6 The Simulation Results

Picture (a)-(e) are single-pole magnetic structure, picture (f) is bipolar magnetic structure. Bipolar magnetic structure can reduce the volume and weight of electromagnetic piston and improve the utilization of permeable material.

\section{Conclusion}

Based on the analysis of simulation results, we can draw the following conclusions:

(1) Increasing the thickness of the cylinder which can reduce the maximum magnetic field intensity of the magnetic circuit, at the same time, the magnetic field distribution of the damping channel may become uniform;

(2) The loading excitation current strength increases, the damping force generated by MR damper increases;

(3) Work space decreases, the magnetic field intensity damping within the channel can greatly improve;

(4) The channel length of the damper is reduced, the magnetic field intensity within the damping channel can be increase;

(5) Permeability of the outer tube material increases, the magnetic field intensity damping within the channel also increases.

(6) Using a two-stage magnetic circuit excitation current load range can be increased to improve the magnetic circuit performance, optimization of the magnetic circuit; After comparing the different winding ways of the dual-stage magnetic circuit, the performance of the opposite winding way is better than the same winding way.

(7) In order to reduce the volume and weight of electromagnetic piston and improve the utilization of magnetic materials, multi-stage magnetic structure shoud be used.

(8) In order to reduce magnetic flux leakage, if the electromagnetic piston outside diameter and shaft length allowed, the magnetic circuit stages should be reduced.

\section{References}

[1] Shulman, Z. P. et a1. Strucature Physical Properties and Dynamics of Magneto-rheological

Suspensions, Int. J. Mutiphase Flow， 1986， 12(6):935-955.

[2] Ribakov Y,Gluck J.Selective controlled base isolation system with Magneto-rheological dampers[J].Earthquake Engineering and Structural Dynamics,2002,31:1301-1324.

[3] Nam Y J, Park M A. Performance evaluation of two different bypass-type MR shock dampers[J]. Journal of Intelligient Material Systems and Structures. 2007, 18(7):707-717. 